\title{
The analysis of whole-body bone scan images by using quantitative improvement in brightness
}

\author{
Mahmoud JALILI ${ }^{1}$, Omid SHAHINMEHR ${ }^{2 *}$, Abdollah CHALECHALE ${ }^{3}$ \\ ${ }^{1}$ Department of Radiology, Faculty of Medicine, University of Medical Sciences and Health \\ Services, Kermanshah, Iran \\ ${ }^{2}$ Department of Computer Engineering, Faculty of Engineering, Kermanshah Branch, Islamic \\ Azad University, Kermanshah, Iran \\ ${ }^{3}$ Department of Computer Engineering, Faculty of Engineering, Razi University, \\ Kermanshah, Iran.
}

\begin{abstract}
Today's, the image processing applications are obvious in the various sciences. The medical image processing has achieved to acceptable results in the field of diagnosing disease. One of these applications which is discussed in this paper is diagnosis injury in whole body bone scan. Whole body bone scan has some applications in the diagnosis of benign lesions such as cardiovascular disease and metabolic bone and sports injuries and also to investigate the pervasiveness of bone. One of the main criteria to diagnose complications in the body is area with high brightness to the brightness of adjacent areas in the image or asymmetry of the brightness in the two-sided areas of the body. In this study, a software has been implemented about the detection of disease-suspected areas that strategies to increase the pixel intensity have been considered to make easier the image segmentation and innovative methods of researcher have been implemented in image preparation to convert to binary mode and find suspected areas. In addition, the software automatically creates reports, this feature will be a big contribution to the doctor. Software assessment has been done on 11 whole body bone scan images and provided the results to the physician, the software succeeded to find the 41 correct areas from 44 injured areas, $93.18 \%$ of cases have been successfully identified. In terms of the number of errors in the areas of diagnosis, 30 cases were observed in 11 images which shows an average of 2.72 errors on each image.
\end{abstract}

Keywords: image processing, medical images, medical diagnostics, nuclear medicine.

\footnotetext{
* Electronic address: omidshahinmehr@gmail.com
} 


\section{Introduction}

In this study, the evaluation of computer application in helping medical diagnosis has been conducted by using image processing extracted from bone scan by nuclear medicine.

Whole body bone scan has some applications in the diagnosis of benign lesions such as cardiovascular disease and metabolic bone and sports injuries and also to investigate the pervasiveness of bone. Bone imaging is performed about three to four hours after injection of radiotracer which is properly absorbed by the body bones. The smallest change in the bone which is caused by various diseases and lesions, such as hidden fractures, tumors and infections can be detected by bone scan before gets appeared on X-ray. Usually imaging is done in anterior and posterior views Figure 1. But sometimes it is necessary that the visible lesions in specific areas scanned from other angles or Single Photon Emission Computed Tomography (SPECT) used Figure 2.

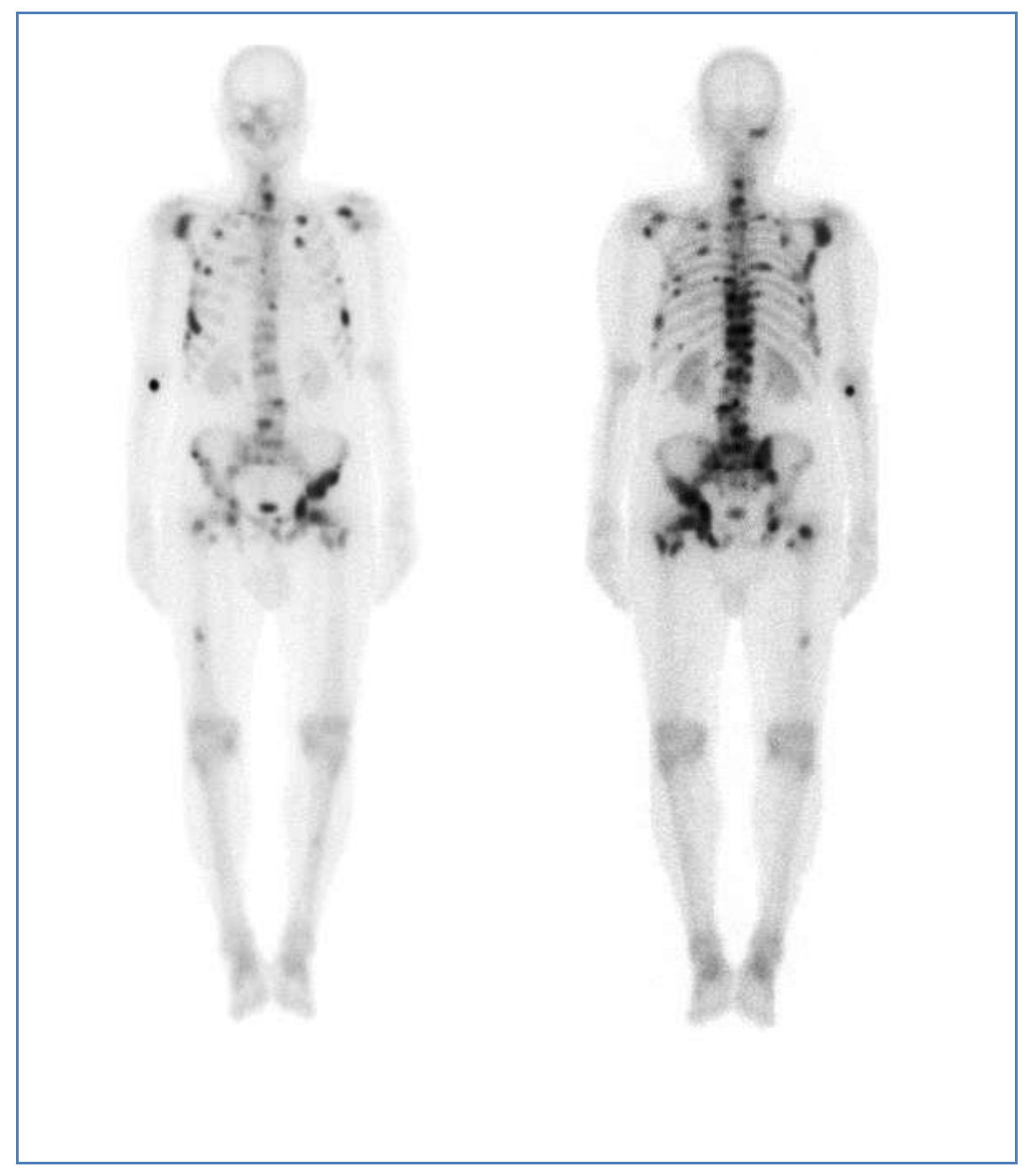

Fig. 1: Anterior view (left) and posterior (right) of whole body bone scan 


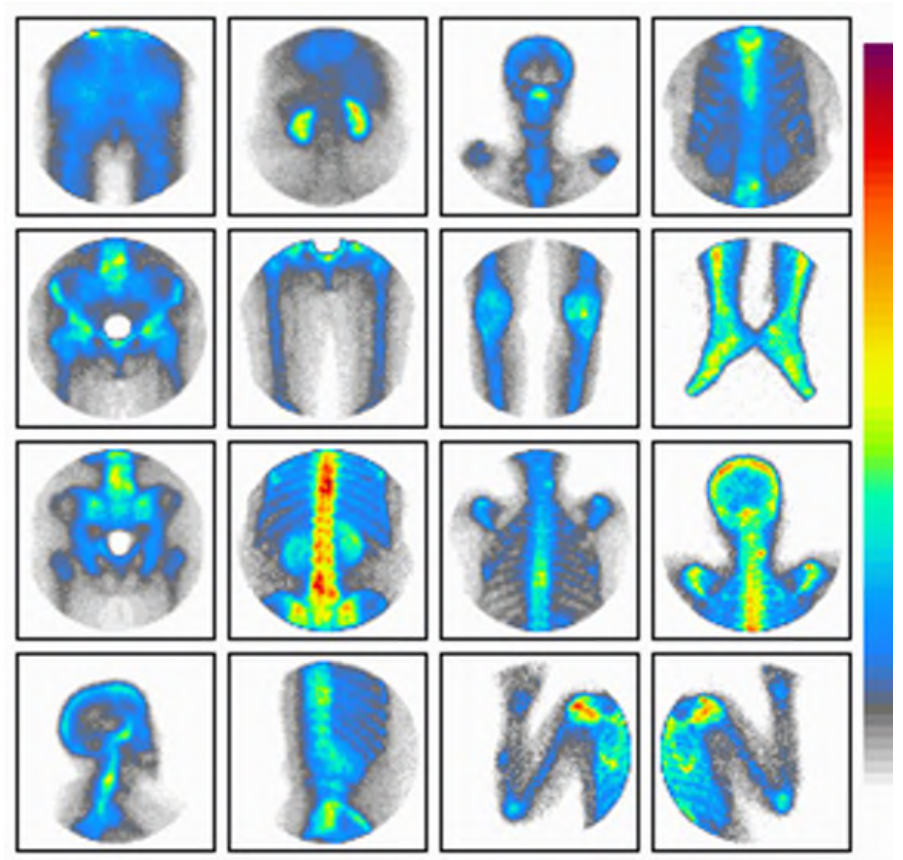

Fig. 2: Scanning views and different angles

Finding injured areas or areas with lesion usually done by doctor eye control. And in cases such as fatigue, multiplicity of diseases, etc., the doctor accuracy is wrong in image viewing and lesion diagnosis. So, designing a system processes the images and identifies suspected areas to help doctors to see images more carefully and make the right decision is necessary.

\section{The problem solving method}

Areas with high brightness in the image to adjacent areas or asymmetry of the brightness in two sides of body is one the main criteria in diagnosing areas suspected to complications in the body. The general approach to process these images and obtain suspicious areas to complications, is that first the body image is segmented to major organs and then the segmentations are processed to find areas with higher brightness to adjacent areas within the segmentation, as well as asymmetry with the same organ on the opposite side of the body.

It should be noted that the purpose of the area is not a pixel with high brightness, but is a set of pixels with high brightness.

The determination of normal brightness also is different according to the type of device and output images and should be set as an input parameter for the system. The normal brightness level is called the threshold. 


\section{A review}

So far, various tasks have been done in the field of image processing, and image segmentation [1] and feature extraction techniques [2] with fuzzy logic or neural network [3] have been used in most methods. Two articles of Chiu and Haung have more references that others and have done a better job so we have chosen it to investigate.

The proposed methods have acceptable results in determining injured areas and slightly act better that algorithms [2].Algorithms [1] is much weaker than algorithm [2] in diagnosing errors. But in the end both systems with a good percentage of correct diagnosis, can be used as a suitable assistant to guide the physician in the diagnosis of disease. By using the results of the studies, the need to implement an optimal way with the low error rate was identified and adding the report writing using expert system design methods also should be considered.

\section{The proposed method}

Due to the poor quality of images and improve quality and reduce noise, some preprocessing is done on image to process whole body bone scan images and obtain the areas suspected to disease. Then body image is segmented to main organs and finally these segmentations are processed to find areas with higher brightness than surrounding areas within the segmentation, as well as asymmetry is processed by the same organ on the other side of body. In next step, the areas detected by the system are marked on image and considering to injury position, a report is provided to doctor from imaging, Figure 3. The used tool to implement is MATLAB. 
Bulletin de la Société Royale des Sciences de Liège, Vol. 85, 2016, p. 952 - 966

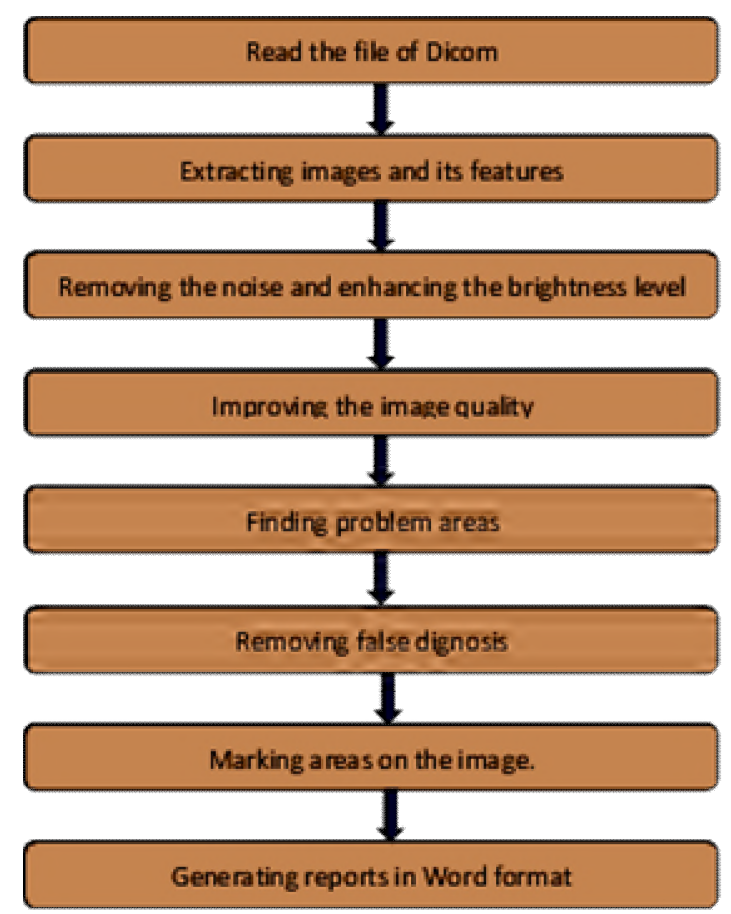

Fig. 3: Block diagram of the steps

The home page of designed program is shown in Figure 4 that the user selects the desired file of Dicom, after selecting the file, the desired image of file information is extracted and displayed to the user.

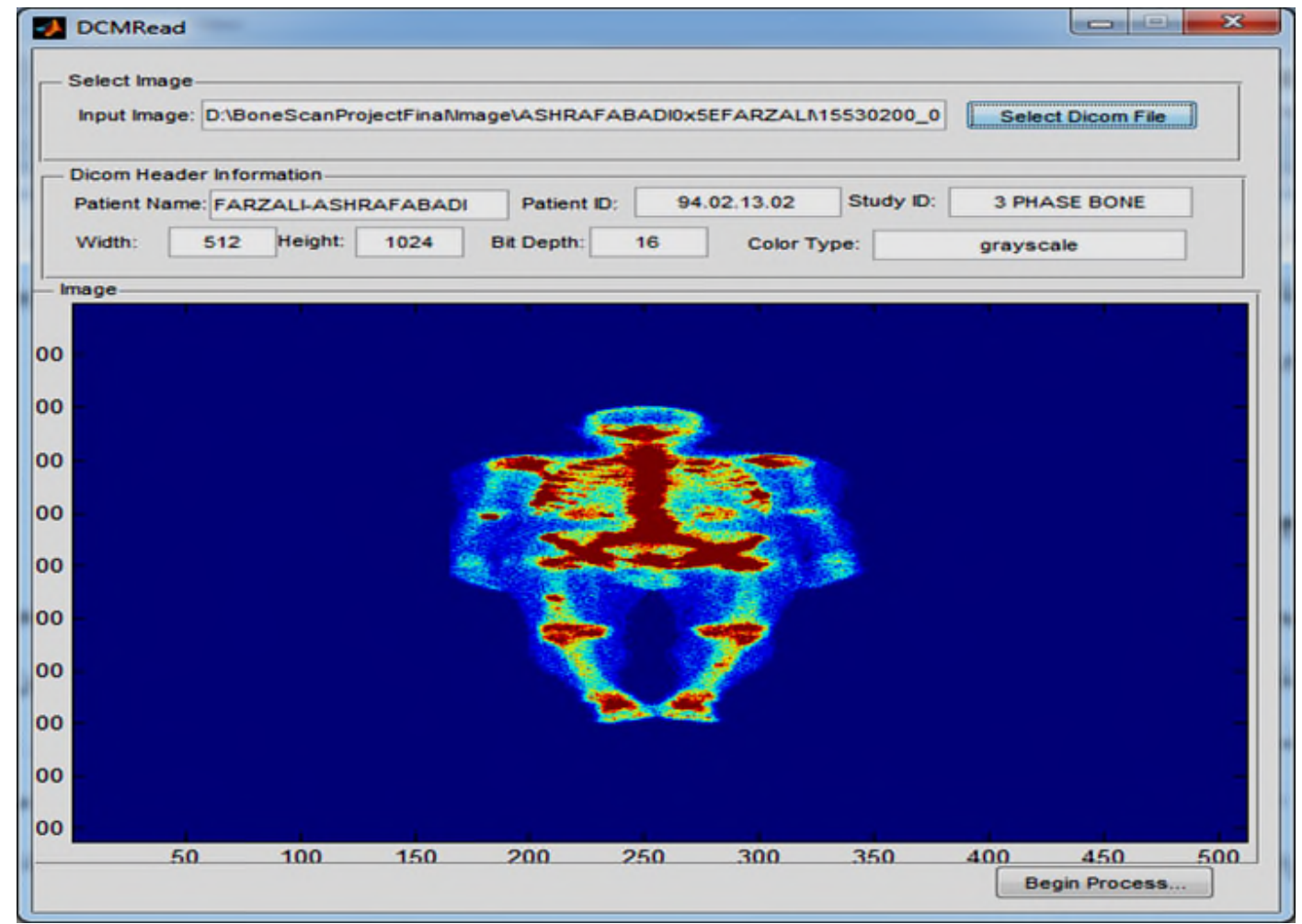

Fig. 4: The program home page 
Due to particular case of bone scan images and after applying various filters on an image, we selected the average mask that is a common mask to remove noise and give image the blur mode. The amount of blueness depends on the size of the mask. Below are examples of images after applying various filters.

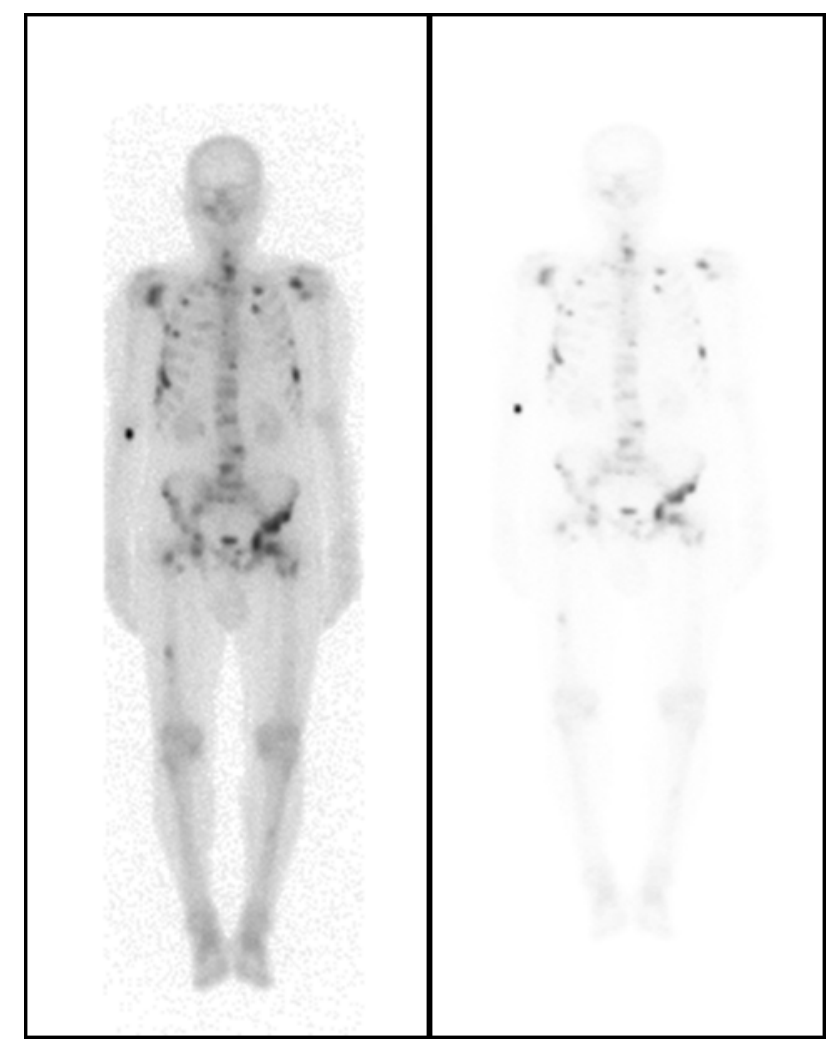

Fig. 5: The original left and right images after applying a the average filter by using mask $3 \times 3$ According to the nature of bone scan images and low quality of images and considering that brightness level (radiopharmaceutical uptake) is different in different organs, common and standard methods do not provide desired outputs to continue processing and detect the areas disease suspected. Therefore, the change in the amount of pixel intensity must be done before starting to image segmentation. According to a survey that was conducted on different images, equation 1 is obtained to increase the amount of pixel intensity which is described in Table 1.

$\mathrm{j}(\mathrm{x}, \mathrm{y})=\left\{\begin{array}{lrr}0, & i(x, y)<M I I \\ i(x, y)+T, & M I I \leq i(x, y) \leq M A I \text { and } & i(x, y)+T \leq 1 \\ 1, & i(x, y)>M A I \text { or } & i(x, y)+T>1\end{array}\right.$

$\mathrm{MII}=(\mathrm{T} * 2)-\mathrm{SD}$ 
Bulletin de la Société Royale des Sciences de Liège, Vol. 85, 2016, p. 952 - 966

Table 1: The formula description of increase in the intensity of image pixels

\begin{tabular}{cc}
\hline Title & Description \\
\hline MAI & The greatest amount of intensity in the image \\
MII & The lowest acceptable intensity in the image \\
T & The image threshold \\
SD & The standard deviation of the intensity of image pixels \\
$\mathbf{j}$ & The intensity of the output image \\
$\mathbf{i}$ & The intensity of the output image \\
\hline
\end{tabular}

First we obtain the lowest acceptable intensity (the amount of intensity that is seen as a suspected area in the image) by using image threshold value that is obtained by standard methods otsu and the standard deviation of image pixels according to equation 1 then we obtain the new intensity value of pixel by using the terms defined in the formula. Below figures show the comparison of image output after implementing above formula and the standard method of MALTAB and a method that does not do any processes on image which better performance in separating and identifying areas is evident by the proposed formula.

Binary image after pre-processing on output image of average filter is shown on Figure 6 that the left image shows no processing in knees, ankles and some areas of the spine and does not show image intensity. The middle image shows binary image using the stretchlim function, this function automatically determines the intensity. By this function, it is observed that there is very weak performance in the diagnosis areas, its difference with the previous method is completely clear. The right image shows binary image which is obtained by implementation of proposed method, the areas are well separated in this image and even a small amount of drug absorption is observed in left tight, the image shows the acceptable performance the method. 
Bulletin de la Société Royale des Sciences de Liège, Vol. 85, 2016, p. 952 - 966
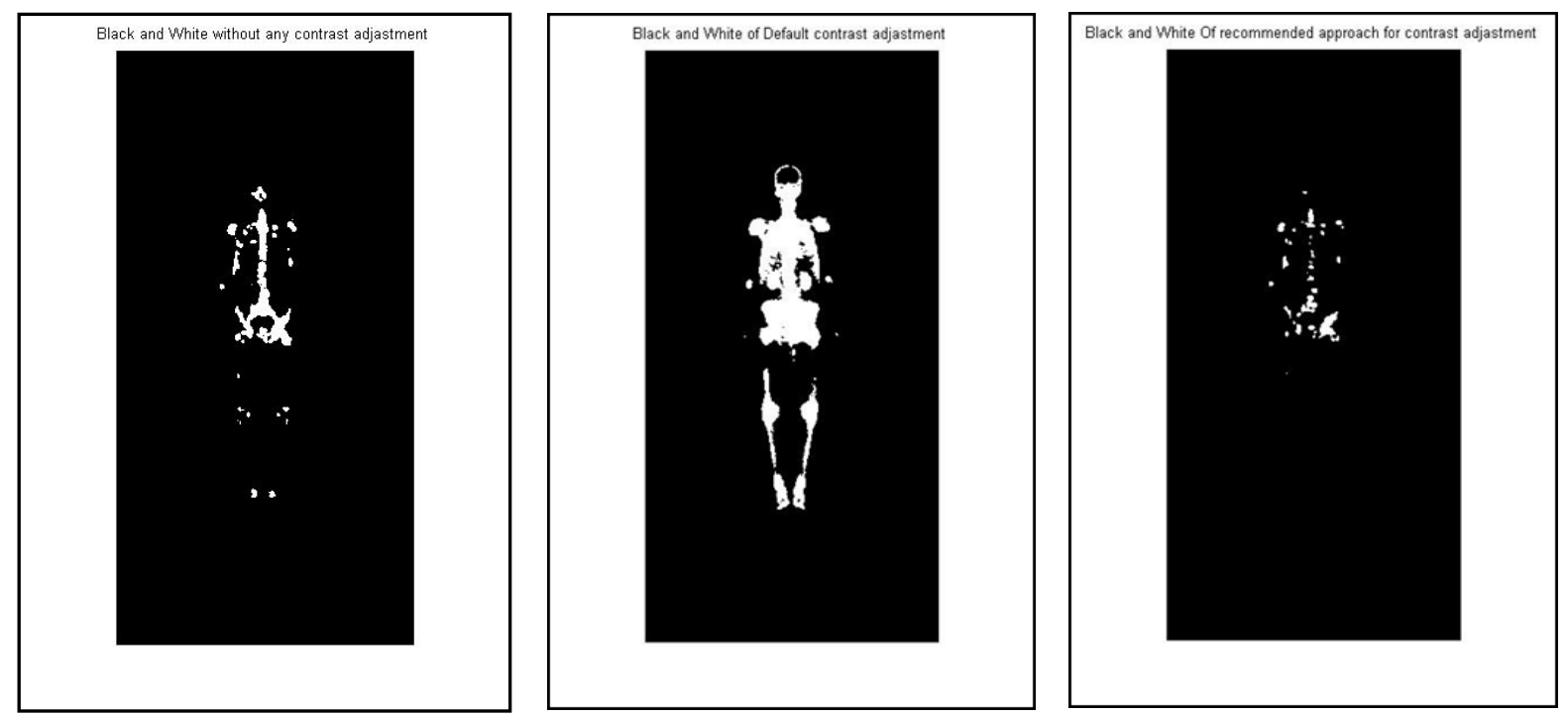

Fig 6: Binary image after implementing changes in the intensity of pixels, left image without change, the middle image the intensity change using stretchlim function of MATLAB, right image the implementation of intensity changes by using the proposed method.

Then, the segregated areas center in the previous step can be obtained by using MATLAB regionprops function and marked by using the obtained coordinates on the original image.

The areas of misdiagnosis must be removed based on the principle of symmetry in Figure 7 , compared to the posterior image after obtaining coordinates of suspected areas and the remaining areas on the image must be marked, Figure 8. It should be noted that the principle of symmetry is not established in the spinal cord and the comparison would be done to up and down vertebrae. 
Bulletin de la Société Royale des Sciences de Liège, Vol. 85, 2016, p. 952 - 966

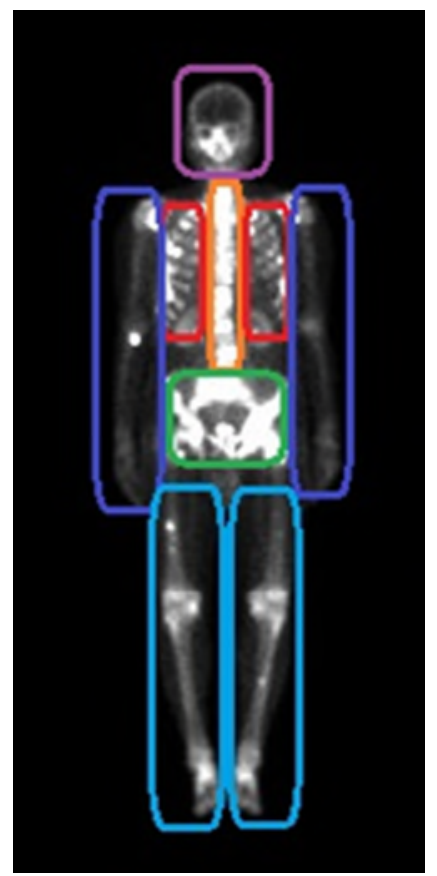

Fig. 7: Bodys segmentation based on symmetry

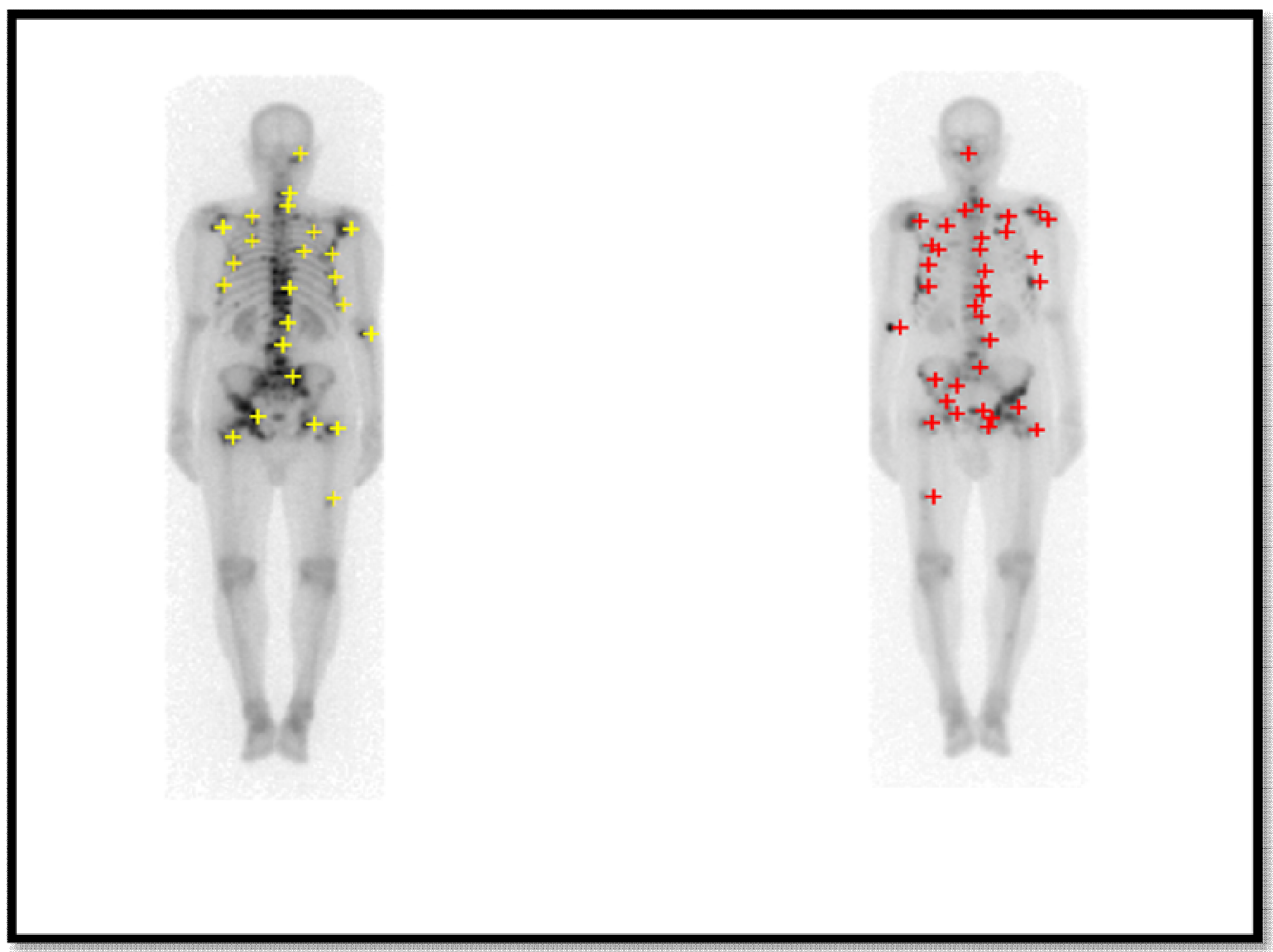

Fig. 8: Removing errors (right image: early diagnosis, left image: final mark)

The provided program has some facilities to provide effective help automatically which will be effective help to the doctor. This program provides a report in a Word format by using information in the dicom file image and based on diagnosed areas which an example of the 
Bulletin de la Société Royale des Sciences de Liège, Vol. 85, 2016, p. 952 - 966

output can be seen in Figure 9. The facilities, means the preparation of a written report from the image data is one of the innovations in the present study.

\section{WHOLE BODY BONE SCAN}

I thest hroj"

S.
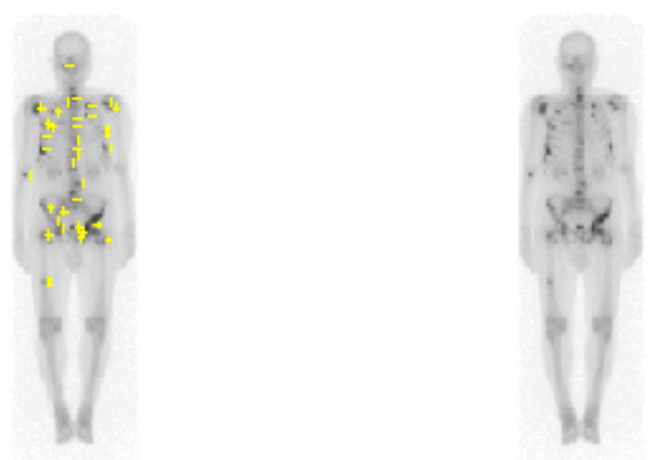

Patient:FARZALI-ASHRAFABADI

In patient bone scan images, below area are suspected to diseases.

Head

Right hand

Right chest

Left chest

Right thigh

Pelvis

Spinal cord

Fig. 9: Sample report in the form of Word file 


\section{The assessment of the proposed method effectiveness}

The 11 patients whole body bone scan images of the nuclear medicine center of kowsar kermanshah are used to assess which are made by a gamma camera, Philips Forte model. Then, the evaluation of software implementation results and next comparing them to doctor ideas and finally comparing them to Chiu and Haung will be done.

After obtaining the software output, the results and used images were presented to kowsar nuclear medicine physicians and technicians and the doctor diagnosis was taken to compare the accuracy of the software. In the following comparison table, the doctor diagnosis and software diagnosis can be seen that the software succeeded to find 41 areas of total 44 , which the $93.18 \%$ of cases successfully identified. 30 cases were observed in 11 areas in the term of the number of errors, we have an average of 2.72 numbers on each image. And finally the software did not diagnose the 3 injured areas.

Table 2: Algorithm test results of 11 images and comparing it with the doctor

\begin{tabular}{cccccc}
\hline $\begin{array}{c}\text { The image } \\
\text { number }\end{array}$ & $\begin{array}{c}\text { The number } \\
\text { of software } \\
\text { diagnoses }\end{array}$ & $\begin{array}{c}\text { The } \\
\text { number } \\
\text { of doctor } \\
\text { diagnoses }\end{array}$ & $\begin{array}{c}\text { The } \\
\text { number } \\
\text { of correct } \\
\text { diagnoses }\end{array}$ & $\begin{array}{c}\text { Number of } \\
\text { wrong } \\
\text { diagnoses }\end{array}$ & $\begin{array}{c}\text { The number of } \\
\text { missed diagnoses }\end{array}$ \\
\hline 1 & 0 & 0 & 0 & 0 & 0 \\
2 & 4 & 2 & 2 & 2 & 0 \\
3 & 1 & 1 & 1 & 0 & 0 \\
4 & 5 & 2 & 2 & 3 & 0 \\
5 & 10 & 9 & 7 & 3 & 2 \\
6 & 7 & 3 & 3 & 4 & 0 \\
7 & 6 & 2 & 2 & 4 & 0 \\
8 & 9 & 5 & 4 & 5 & 0 \\
9 & 4 & 1 & 1 & 3 & 0 \\
10 & 0 & 0 & 0 & 0 & 0 \\
11 & 25 & 19 & 19 & 6 & $\mathbf{3}$ \\
\hline Total & $\mathbf{7 1}$ & $\mathbf{4 4}$ & $\mathbf{4 1}$ & $\mathbf{3 0}$ & $\mathbf{0 . 2 7}$ \\
\hline Average & $\mathbf{6 . 4 6}$ & $\mathbf{4}$ & $\mathbf{3 . 7 3}$ & $\mathbf{2 . 7 2}$ & \\
\hline
\end{tabular}




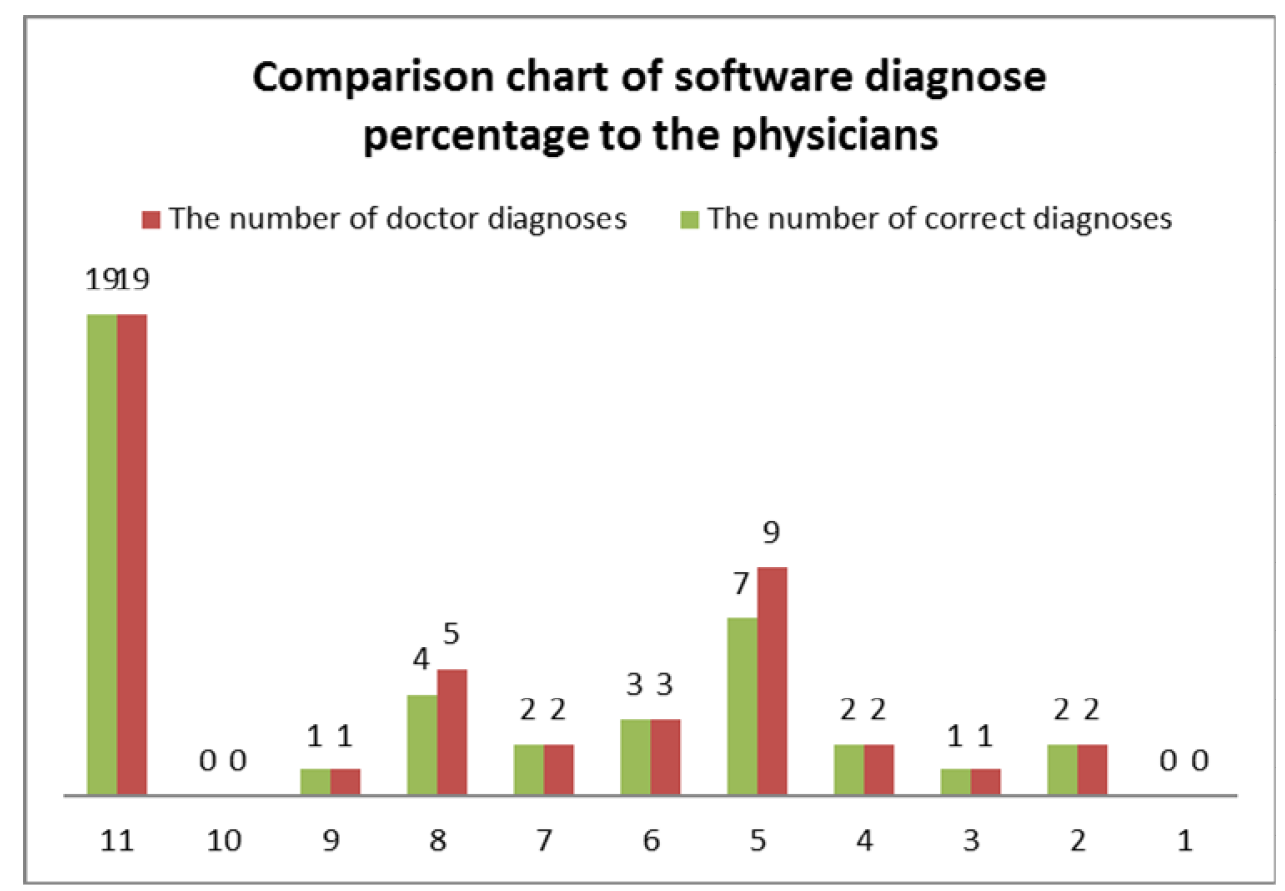

Fig. 10: Comparison chart of the software diagnosis number and physicians

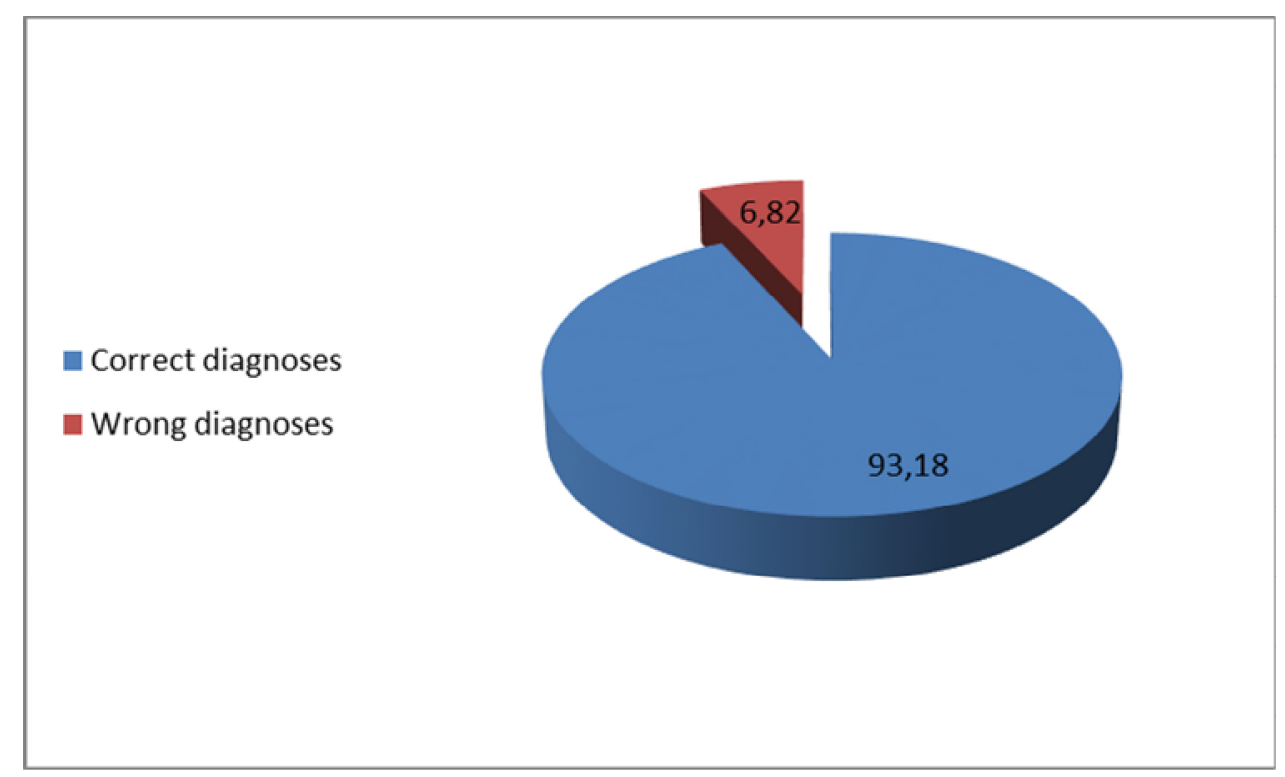

Fig. 11: Comparison chart of software diagnose percentage to the physicians

Then, the proposed method was compared to Chiu Yin algorithm and Huang, Kao and Chen algorithm. That the proposed method in terms of the percentage of correct diagnosis showed 93.18\% better performance than the two methods presented above. It also had better performance than other algorithms in the diagnosing errors with an average value of about 2.72 per image. The proposed algorithm by Yin and Chiu with an average of 34 misdiagnoses 
had much weaker performance than the other two methods. Comparison charts are shown in Figs. 12 and 13.

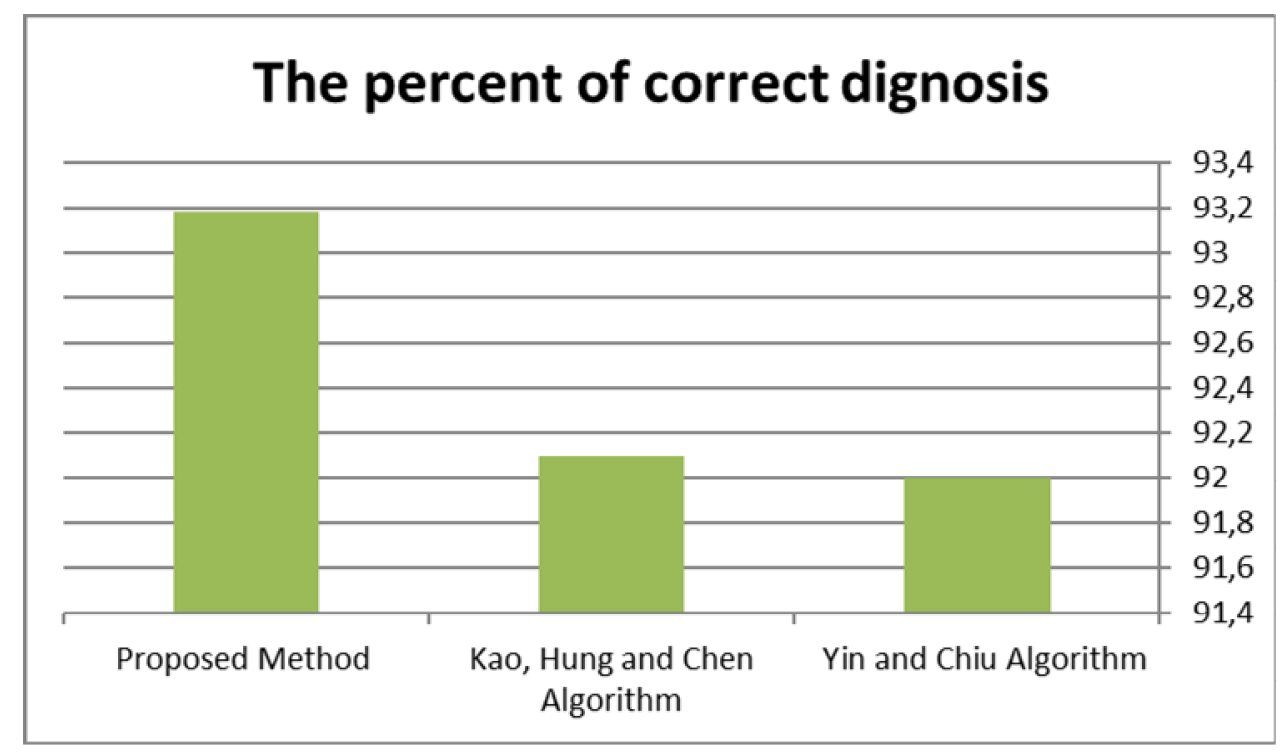

Fig. 12: The comparison chart of percentage of proposed method correct diagnosis with Yin and Chiu algorithm, Huang, Kao and Chen algorithm

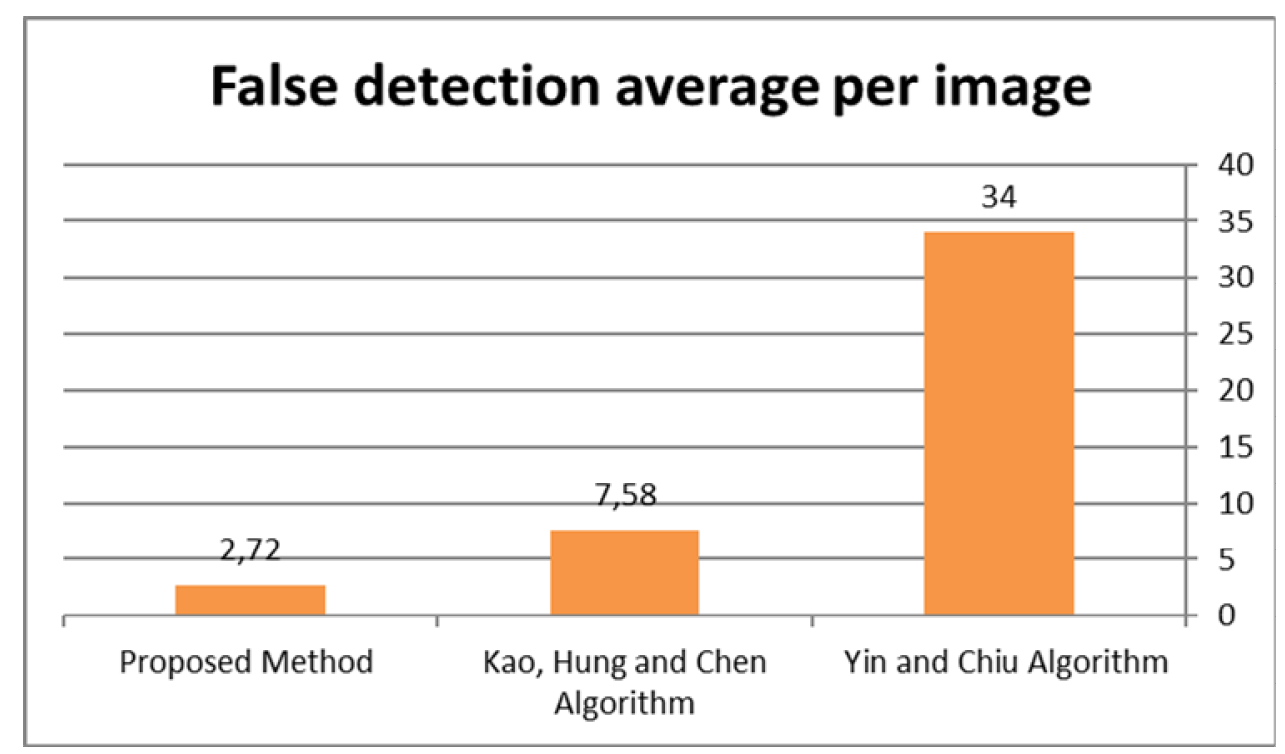

Fig. 13: The comparison chart of proposed method false detection average with Yin and Chiu algorithm and Huang، Kao and Chen algorithm 


\section{Conclusion and Future Work}

In this study, the design of a whole body bone scan image processing system which is able to detect determine the suspected areas in the image, has been studied and implemented. This system, in addition to mark these areas on the image, generates the answer of scan report which is extracted from Dicom file by determining the type and characteristics of the patient. The main focus is on the amount of pixels intensity increase innovative methods of researcher have been implemented in image preparation to convert to binary mode and find suspected areas. Software assessment has been done on 11 whole body bone scan images and provide the results to the physician, the software succeeded to find the 41 correct areas from 44 injured areas which $93.18 \%$ of cases have been successfully identified. In terms of the number of errors in the areas of diagnosis, 30 cases were observed in 11 images which shows an average of 2.72 errors on each image.In total, the proposed algorithm has the better performance compared to existing methods of problem solving. And also shows that the proper pre-processing performed on the images before performing the segmentation is the most important criteria in increasing the efficiency of image processing software. Reviews and providing guidelines to improve the performance of the algorithm can be mentioned as future work and proposals as follows:

- The increase of test images number

- Reducing the number of detected errors using new algorithms implementation or improving existing methods.

- Increasing the number of correct diagnoses using new algorithms implementation or improving existing methods.

- Work on the asymmetry images in which the patient has organ failure or poor body image is a tilt and asymmetry.

- Certified system design according to the type of abnormal organ, age and sex and other effective conditions and parameters in the diagnosis of the disease and also detecting and reporting diseases to send to doctor. 


\section{References}

1. T. K. Yin and N, T. Chiu, "A computer-aided diagnosis for locating abnormalities in bone scintigraphy by a fuzzy system with a threestep minimization approach" IEEE Transactions on Nuclear Science, vol. 23, no. 5, May 2004.

2. J. Y. Huang, P. F. Kao, Y. S. Chen, "A Set of Image Processing Algorithms for ComputerAided Diagnosis in Nuclear Medicine Whole Body Bone Scan Images." IEEE Transactions on Nuclear Science vol. 54, no. 3, June 2007.

3. L. Sajn, M. Z. Kukar, I. Kononenko, M. M. Cinski. "Computerized segmentation of wholebody bone scintigrams and its use in automated diagnostics", Elsevier Computer Methods and Programs in Biomedicine 80, 47-55, March 2005.

4. J. Y. Huang, M. F. Tsai, P.- F. Kao, Y. S. Chen, "Automatic Computer-aided SacroiliacJoint Index Analysis for Bone Scintigraphy.", Elsevier Computer Methods And Programs in Biomedicine 98, 15-26 ,July 2010.

5. M. Lyra, A. Ploussi, A. Georgantzoglou, "Matlab As a tools in Nuclear medicine image processing,"http://www.intechopen.com/books/matlab-a-ubiquitous-tool-for-the-practicalengineer/matlab-as-a-tool-in-nuclear-medicine-image-processing, Visited on Oct 2015.

6. R. C. Gonzalez, R. E. Woods, S. L. Eddins, Digital Image Processing Using MATLAB: Gatesmark Publishing 2009. 